\title{
Modeling second-harmonic generation by use of mode expansion
}

\author{
Björn Maes, Peter Bienstman, and Roel Baets \\ Department of Information Technology, Ghent University-Interuniversity Micro-Electronics Center, \\ St.-Pietersnieuwstraat 41, 9000 Ghent, Belgium
}

Received October 20, 2004; revised manuscript received February 15, 2005; accepted April 4, 2005

\begin{abstract}
We present an accurate and efficient method of modeling second-harmonic generation in two-dimensional structures by use of eigenmode expansion. By using the undepleted-pump approximation we uncouple the calculations for the fundamental and second harmonic. Expansion of the field in eigenmodes gives rise to a linear matrix formalism. The method includes reflections and is especially suited for periodic structures. Several examples, including a two-dimensional photonic-crystal-cavity device, are studied. (C) 2005 Optical Society of America

OCIS codes: $190.2620,190.4420,230.4320$.
\end{abstract}

\section{INTRODUCTION}

In future all-optical wavelength-division multiplexing networks, nonlinearities will necessarily play an important role in switches or wavelength converters. One of the simplest schemes for the latter is second-harmonic generation (SHG). To design efficient, integrated SHG components new numerical tools are needed.

Many studies of SHG examine the plane-wave or onedimensional situation, where analytical insight can be gained into the processes in periodic structures or devices doped with a defect. ${ }^{1,2}$ Most of the calculation methods therefore focus on a single-mode approach. The situation in two dimensions is less explored. ${ }^{3-7}$ Here transverse effects need to be addressed. In high-contrast waveguides with air slits, for example, radiation losses must be controlled. ${ }^{3}$ Interplay between different transverse modes in parallel waveguides can increase SHG because of phase matching. ${ }^{4}$ Photonic-crystal waveguide modes can also be examined for SHG opportunities. ${ }^{5}$

The aim of this paper is to introduce an extension to the eigenmode expansion method for SHG in twodimensional dielectric structures. The proposed method is fully vectorial and can handle radiation losses. We apply the undepleted-pump approximation to construct a linear matrix formalism. With this formalism the second harmonic is calculated in one run. The method is especially efficient for periodic structures, because data can be reused.

We describe the simulation method in Section 2. After a short overview of the linear eigenmode method, the introduction of the SHG extension follows. Validation of the algorithm is provided by a waveguide example in Section 3 and a transversely periodic structure in Section 4. A more advanced photonic-crystal-cavity device is discussed in Section 5 .

\section{MODELING TECHNIQUE}

\section{A. Linear Mode Expansion}

In the linear mode expansion method one chooses a main propagation direction and divides the structure into sections invariant along this direction. An example of a simple structure with three parts is shown in Fig. 1. The forward propagating field in such an invariant section can be described as a superposition of eigenmodes:

$$
\begin{gathered}
\mathbf{E}(\mathbf{r})=\sum_{i=1}^{N} F_{i}^{\prime} \exp \left(-j \beta_{i} z\right) \mathbf{E}_{i}\left(\mathbf{r}_{\mathrm{t}}\right)=\sum_{i} F_{i} \mathbf{E}_{i}\left(\mathbf{r}_{\mathrm{t}}\right), \\
\mathbf{H}(\mathbf{r})=\sum_{i} F_{i}^{\prime} \exp \left(-j \beta_{i} z\right) \mathbf{H}_{i}\left(\mathbf{r}_{\mathrm{t}}\right)=\sum_{i} F_{i} \mathbf{H}_{i}\left(\mathbf{r}_{\mathrm{t}}\right),
\end{gathered}
$$

with $\mathbf{r}_{\mathrm{t}}$ the transverse component of $\mathbf{r} . N$ is the number of modes included in the calculations. Similar expressions can be derived for the backward-propagating field with mode amplitudes $B_{i}$. The mode profiles $\mathbf{E}_{i}$ and $\mathbf{H}_{i}$ and their corresponding propagation constants $\beta_{i}$ are determined from the transversal index profile. The eigenmodes of a section are solutions of the following eigenvalue equation:

$$
\nabla_{\mathrm{t}}^{2} \mathbf{E}+k^{2} \mathbf{E}=\beta^{2} \mathbf{E},
$$

with $\nabla_{\mathrm{t}}^{2}$ the transverse Laplacian and $k$ the wave number. These solutions form an orthogonal set. They can be normalized, and in our formalism this is expressed as

$$
\int_{t}\left(\mathbf{E}_{i} \times \mathbf{H}_{j}\right) \cdot \mathbf{u}_{z} \mathrm{~d} l=\delta_{i j},
$$

with $\mathbf{u}_{z}$ the unit vector along $z, \delta_{i j}$ the Kronecker delta, and integration along the transverse direction. We denote this integral as $\left\langle E_{i} \mid H_{j}\right\rangle$, where we understand that only 


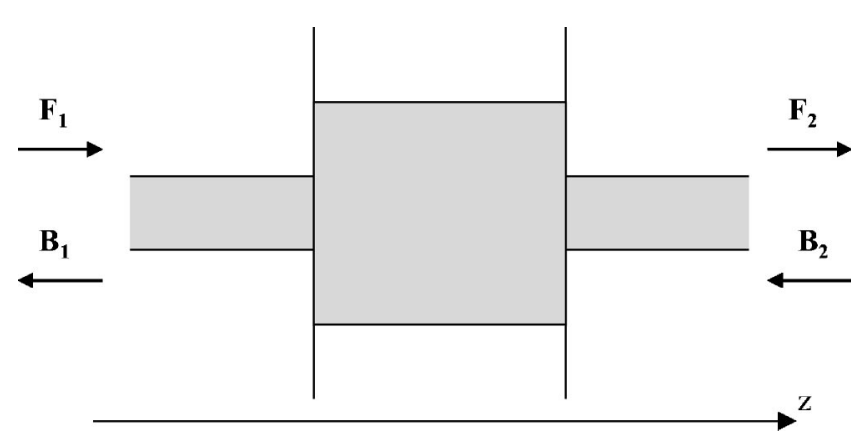

Fig. 1. Structure with three invariant sections along $z$. Mode amplitude vectors at input and output are indicated.

the transverse components are needed. Finally, after calculation of the modes, the field in a section can be reduced to a vector of complex mode amplitudes $[\mathbf{F}, \mathbf{B}]$.

To combine different sections the well-known modematching technique is used. This technique imposes continuity of the tangential total field components to infer transmission and reflection matrices for the mode amplitudes on the interface between two sections. In the end we combine these interface matrices with the propagation constants to derive a scattering matrix $\mathbf{S}$ for the total structure. The behavior of the structure in Fig. 1 is thus described by

$$
\left[\begin{array}{l}
\mathbf{F}_{2} \\
\mathbf{B}_{1}
\end{array}\right]=\mathbf{S} \cdot\left[\begin{array}{l}
\mathbf{F}_{1} \\
\mathbf{B}_{2}
\end{array}\right]=\left[\begin{array}{ll}
\mathbf{T}_{12} & \mathbf{R}_{21} \\
\mathbf{R}_{12} & \mathbf{T}_{21}
\end{array}\right] \cdot\left[\begin{array}{l}
\mathbf{F}_{1} \\
\mathbf{B}_{2}
\end{array}\right],
$$

with $\left[\mathbf{F}_{1}, \mathbf{B}_{2}\right]$ the input and $\left[\mathbf{F}_{2}, \mathbf{B}_{1}\right]$ the output mode amplitude vectors. More information regarding the implementation of the linear mode expansion method can be found in Ref. 8.

\section{B. Extension for Second-Harmonic Generation}

Here we derive how this matrix formalism changes if we include SHG. Note that we have already extended eigenmode expansion for the Kerr nonlinearity, ${ }^{9}$ which highlights the versatility of this scheme. The SHG method is an extension of a plane-wave formalism combined with the eigenmode method. ${ }^{10}$ We start from the adjusted Hemholtz equations in the case of SHG: ${ }^{11}$

$$
\begin{aligned}
\nabla^{2} E_{\omega}+k_{\omega}^{2} E_{\omega} & =-\omega^{2} \mu_{0} \epsilon_{0} 2 d_{\mathrm{nl}} E_{2 \omega} E_{\omega}^{*}, \\
\nabla^{2} E_{2 \omega}+k_{2 \omega}^{2} E_{2 \omega} & =-(2 \omega)^{2} \mu_{0} \epsilon_{0} d_{\mathrm{nl}} E_{\omega}^{2},
\end{aligned}
$$

with $E_{\omega}$ and $E_{2 \omega}$ the complex fields of the fundamental and second harmonic, respectively. $\nabla^{2}$ is the total Laplacian, and $k_{\omega}$ and $k_{2 \omega}$ denote the appropriate wave numbers.

In the undepleted-pump approximation we ignore the right-hand side of Eq. (5). With this approximation we can linearly solve the fundamental fields with the methods of Subsection 2.A. ${ }^{12}$ Note that a high pump depletion is seldom reached in wavelength-scale integrated structures.

The formalism is presented here for generation from TM to TM (one $E$ component of which is out-of-plane) with a single nonlinear polarization component along the outof-plane direction $y$. However, the adaptation to other polarizations is straightforward. Indeed, from the vectorial Helmholtz equation

$$
\nabla^{2} \mathbf{E}_{2 \omega}+k_{2 \omega}^{2} \mathbf{E}_{2 \omega}=-(2 \omega)^{2} \mu_{0} \mathbf{P}_{\mathrm{nl}}
$$

with $\mathbf{P}_{\mathrm{nl}}$ the nonlinear polarization vector, we conclude that $P_{\mathrm{nl}, x}$ and $P_{\mathrm{nl}, z}$ couple to the TE polarization, whereas $P_{\mathrm{nl}, y}$ couples to the TM polarization. This means we have to consider only scalar Helmholtz equations for the appropriate electric field components, as in Eq. (6). The polarizations remain independent. The exact expression for the right-hand side of Eq. (6) depends on the polarization of the fundamental and the nonlinearity tensor. Changing these terms results only in different components being used for the mode overlap integrals, which will become clear below. We employ only one term on the righthand side of Eq. (6) because extra terms are managed by means of superposition of the individual contributions.

To solve Eq. (6) in an invariant section we write the fields as a sum of eigenmodes:

$$
\begin{aligned}
\mathbf{E}_{\omega} & =\sum_{i} A_{i}^{\prime} \exp \left(-j \beta_{\omega, i} z\right) \mathbf{E}_{\omega, i}, \\
\mathbf{E}_{2 \omega} & =\sum_{i} C_{i}^{\prime}(z) \exp \left(-j \beta_{2 \omega, i} z\right) \mathbf{E}_{2 \omega, i}=\sum_{i} C_{i}(z) \mathbf{E}_{2 \omega, i} .
\end{aligned}
$$

We assume that only copropagating modes interact nonlinearly, because of phase mismatch; therefore we can ignore the modes propagating in the other direction for the moment. Note the $z$ dependence of the mode amplitudes $C_{i}^{\prime}$, which indicates the possibility of generation. In the linear case these amplitudes are constant in a section. The constant amplitudes $A_{i}^{\prime}$ are provided by the first linear calculation. Substituting Eqs. (8) into Eq. (6) we get a second-order differential equation. The derivatives of the mode profiles can be eliminated with Eq. (2). We ignore the second-order derivatives of the envelopes $C_{i}^{\prime}(z)$. The resulting relation contains sums over modes. We can select one second-harmonic mode by using the orthonormality of Eq. (3). Performing this operation $\left\langle. \mid H_{2 \omega, i}\right\rangle$ results in the first-order differential equation

$$
\begin{aligned}
\frac{\mathrm{d} C_{i}^{\prime}}{\mathrm{d} z}= & -2 j k_{0}^{2} \sum_{k} A_{k}^{\prime 2} \frac{\exp \left(j \Delta \beta_{k i} z\right)}{\beta_{2 \omega, i}}\left\langle d_{\mathrm{nl}} E_{\omega, k}^{2} \mid H_{2 \omega, i}\right\rangle \\
& -4 j k_{0}^{2} \sum_{l, m} A_{l}^{\prime} A_{m}^{\prime} \frac{\exp \left(j \Delta \beta_{l m i} z\right)}{\beta_{2 \omega, i}}\left\langle d_{\mathrm{nl}} E_{\omega, l} E_{\omega, m} \mid H_{2 \omega, i}\right\rangle,
\end{aligned}
$$

with $\Delta \beta_{k i}=\beta_{2 \omega, i}-2 \beta_{\omega, k}, \quad \Delta \beta_{l m i}=\beta_{2 \omega, i}-\beta_{\omega, l}-\beta_{\omega, m}$, and $k_{0}$ $=\omega / c$. With $\Sigma_{l, m}$ we indicate $\Sigma_{l=1}^{N-1} \sum_{m=l+1}^{N}$. We write the overlap integrals as

$$
\begin{gathered}
O_{k i}=\left\langle d_{n l} E_{\omega, k}^{2} \mid H_{2 \omega, i}\right\rangle, \\
O_{l m i}=\left\langle d_{n l} E_{\omega, l} E_{\omega, m} \mid H_{2 \omega, i}\right\rangle .
\end{gathered}
$$

Equation (9) can be integrated to 


$$
\begin{aligned}
C_{i}^{\prime}(z)= & C_{i}^{\prime}(0)-2 k_{0}{ }^{2} \sum_{k} A_{k}^{\prime 2} \frac{\exp \left(j \Delta \beta_{k i} z\right)-1}{\beta_{2 \omega, i} \Delta \beta_{k i}} O_{k i} \\
& -4 k_{0} \sum_{l, m} A_{l}^{\prime} A_{m}^{\prime} \frac{\exp \left(j \Delta B_{l m i} z\right)-1}{\beta_{2 \omega, i} \Delta \beta_{l m i}} O_{l m i} .
\end{aligned}
$$

The terms on the right side of this equation are physically clear. The first term represents the linear propagation. The second and third terms describe SHG from one fundamental mode to one second-harmonic mode and from two fundamental modes to one second-harmonic mode, respectively. The integrals weigh the overlap of the mode profiles, whereas the factors with $\Delta \beta$ incorporate the modal phase-matching. Note that in the case of perfect phase-matching, or $\Delta \beta=0$, Eq. (9) can still be solved. However, these expressions are not necessary in practice. If one includes dispersion, exact phase-matching is seldom encountered.

The previous analysis allows us to construct a matrix formalism with the inclusion of modes propagating in both directions. Note that we work here with the unprimed mode amplitudes; see Eq. (8). For this convention Eq. (12) can easily be adapted with $C_{i}^{\prime}(z)$ $=C_{i}(z) \exp \left(j \beta_{2 \omega i} z\right)$. By inspecting Eq. (12) we can write the bidirectional propagation of modes in an invariant section as:

$$
\begin{aligned}
& {\left[\begin{array}{l}
\mathbf{F}_{2} \\
\mathbf{B}_{1}
\end{array}\right]} \\
& =\left[\begin{array}{cc}
\boldsymbol{d i a g}\left[\exp \left(-j \beta_{2 \omega, i} L\right)\right] & \mathbf{0} \\
\mathbf{0} & \operatorname{diag}\left[\exp \left(-j \beta_{2 \omega, i} L\right)\right]
\end{array}\right] \cdot\left[\begin{array}{l}
\mathbf{F}_{1} \\
\mathbf{B}_{2}
\end{array}\right] \\
& +\left[\begin{array}{l}
\mathbf{N}_{\mathrm{fw}} \\
\mathbf{N}_{\mathrm{bw}}
\end{array}\right],
\end{aligned}
$$

with diag indicating a diagonal matrix and $L$ the length of the section. In the following we denote $\operatorname{diag}[\exp ($ $\left.\left.-j \beta_{2 \omega, i}\right)\right]$ as $\mathbf{P}(L)$. Positions of the vectors are the same as in Fig 1, with the difference that Eq. (13) describes a structure without interfaces. $\mathbf{N}_{\mathrm{fw}}$ and $\mathbf{N}_{\mathrm{bw}}$ are calculated by Eq. (12) with input from the forward and backward fundamental mode amplitudes, respectively.

We now have to implement interfaces in the algorithm. For this purpose we divide the structure into pieces consisting of an interface followed by a certain length of an invariant section; see Fig. 2. The interface can be described by a scatter matrix of the same form as in Eq. (4), and the section by Eq. (13). Combining these two we easily get

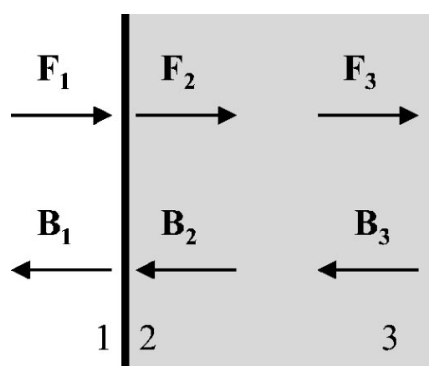

Fig. 2. Interface followed by an invariant section.

$$
\begin{aligned}
{\left[\begin{array}{l}
\mathbf{F}_{3} \\
\mathbf{B}_{1}
\end{array}\right]=} & {\left[\begin{array}{cc}
\mathbf{P}(L) \mathbf{T}_{12} & \mathbf{P}(L) \mathbf{R}_{21} \mathbf{P}(L) \\
\mathbf{R}_{12} & \mathbf{T}_{21} \mathbf{P}(L)
\end{array}\right] \cdot\left[\begin{array}{l}
\mathbf{F}_{1} \\
\mathbf{B}_{3}
\end{array}\right] } \\
& +\left[\begin{array}{c}
\mathbf{P}(L) \mathbf{R}_{21} \mathbf{N}_{\mathrm{bw}}+\mathbf{N}_{\mathrm{fw}} \\
\mathbf{T}_{21} \mathbf{N}_{\mathrm{bw}}
\end{array}\right] .
\end{aligned}
$$

Note that the generated second harmonic is scattered by the interfaces. In the same way as for the previous equation different chunks can be concatenated, which after some algebra results in the same form for an entire structure:

$$
\left[\begin{array}{l}
\mathbf{F}_{\text {out }} \\
\mathbf{B}_{\text {out }}
\end{array}\right]=\mathbf{S} \cdot\left[\begin{array}{l}
\mathbf{F}_{\text {in }} \\
\mathbf{B}_{\text {in }}
\end{array}\right]+\left[\begin{array}{l}
\mathbf{N}_{\text {tot,fw }} \\
\mathbf{N}_{\text {tot }, \text { bw }}
\end{array}\right] .
$$

The scatter matrix $\mathbf{S}$ is the same as in the linear case. The $\mathbf{N}$ vectors immediately give the generated output if there is no external second-harmonic input.

\section{Discussion}

The proposed algorithm has several characteristics of efficiency. First one evaluation of the analytical Eq. (12) is enough to calculate the generation in an invariant section of arbitrary length. There is no need to discretize in the propagation direction, in contrast to the finite-differencetime-domain or beam-propagation methods. One of the main advantages of the linear mode expansion method is thus conserved. Furthermore, for the overlap integrals only the nonlinear sections have to be included. Often this means integration has to be done over a limited transversal part. Nowadays periodic structures are heavily investigated, and our method is especially suited for these devices. Data such as mode profiles, propagation constants, scatter matrices, and overlap integrals can be reused effectively.

Another characteristic is the flexibility of the coupling scheme. It is possible to choose the subsets of fundamental and second-harmonic modes that couple nonlinearly. This happens simply by neglecting the nonlinear terms in Eq. (12) for the excluded modes. Because of phase mismatch, low overlap, or the excitation specifics, many structures can be modeled with only the lower modes contributing nonlinearly. However, higher-order modes are still included in the linear scattering to simulate rigorously effects such as radiation losses.

To determine how many modes are necessary one needs to increase the number of nonlinearly included modes until convergence is reached. There is no general rule, and the same method is used for linear mode expansion calculations. Note that we can drastically reduce the total number of modes included in the eigenmode expansion by employing perfectly-matched layer boundary conditions. ${ }^{8}$

Finally many devices can be understood by way of their underlying modal behavior. Because the proposed method has immediate acces to these data, it is very suitable for interpretation of the structures. This is in contrast with other methods that manipulate only the total fields.

\section{WAVEGUIDE}

We have checked the technique in the plane-wave limit and found good agreement with previously reported re- 


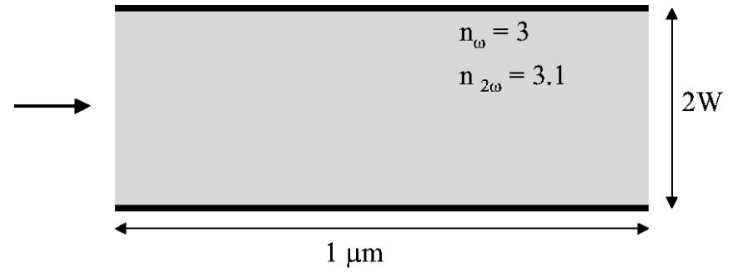

Fig. 3. Geometry of the waveguide example.

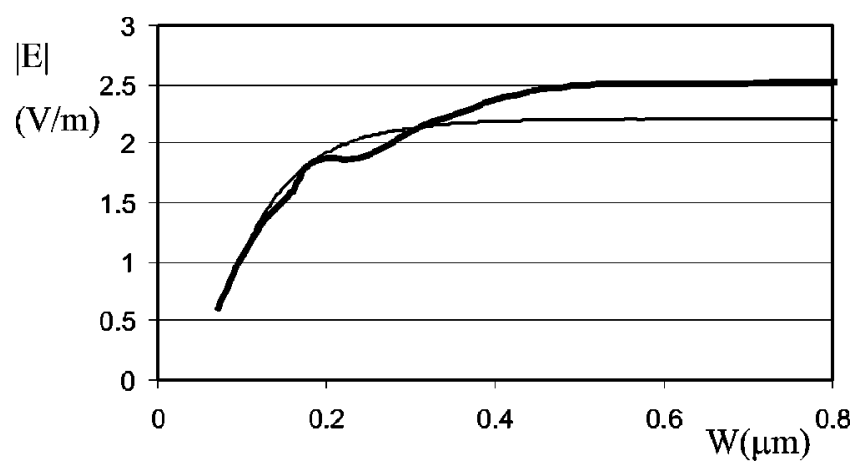

Fig. 4. Generated electric field in the center at the end of the waveguide section, versus half the waveguide width. The light curve is the result with one mode, the heavy curve, with multiple modes.

sults in a layer structure. ${ }^{13}$ Because the method is an extension of a known plane-wave method, we focus instead on a structure that shows the necessity of a multimode approach to obtain more exact results. The proposed example is a waveguide; see Fig. 3. The core indices are 3.0 and 3.1 for fundamental and second harmonic, respectively, whereas the background is air. The waveguide has length $1 \mu \mathrm{m}$ and we operate at $\lambda=1.55 \mu \mathrm{m}$. We assume nonlinear generation from TM to TM with coefficient $d_{\mathrm{nl}}$ $=400 \mathrm{pm} / \mathrm{V}$. The structure is excited with the fundamental ground mode, such that the electric field in the center is $10^{5} \mathrm{~V} / \mathrm{m}$.

We now vary the waveguide width and examine the second-harmonic field at the center of the output position. The calculations are done for one second-harmonic mode and for multiple modes. A maximum of 40 modes is used. Because of the short length the contributions of some radiation modes are needed. Results are shown in Fig. 4. Until a width of $0.132 \mu \mathrm{m}$ both curves agree, and this is precisely where the waveguide becomes multimodal. At $0.8 \mu \mathrm{m}$ there are already seven guided modes, and the field with multiple modes converges toward the planewave value of $2.54 \mathrm{~V} / \mathrm{m}$.

One might expect that the single-mode calculation also converges to the plane-wave case in the limit of broad waveguides. However, in this limit all the modes become plane-wavelike in the center, and their contributions are governed by the overlap integral with the (square of the) fundamental ground mode. An analysis with modes of a waveguide with hard walls (zero field on the boundaries) shows that the overlap integrals of the consecutive modes behave as $O_{00}, O_{00} / 5,-O_{00} / 35, \ldots$. Thus about five modes are enough for accurate results. Clearly this example shows that the interference of the many generated modes has to be taken into account.

\section{TRANSVERSE GRATING}

In this section we simulate a structure proposed in Ref. 4 and depicted in Fig. 5. It consists of a transversely periodic array of waveguides with length $d$ in air excited by a TM plane wave. Because of the finite length $d$, reflections and modes propagating in both directions have to be taken into account.

The grating has a period $\Lambda=0.65 \lambda$ and the waveguides have width $w=0.09 \Lambda$ with indices $n_{\omega}=3.346$ and $n_{2 \omega}$ $=3.539$. We have to include only the box in dotted lines in Fig. 5 for the calculations. Indeed, because of the TM excitation and the symmetry of the structure we can use perfect magnetic walls $(\partial E / \partial x=0)$ for the upper and lower boundaries. Furthermore, because the areas to the left and right of the box are invariant along $z$, we may end the calculations just outside the interfaces.

We compare the forward SHG of this device with the case of bulk nonlinear material of the same thickness $d$ in Fig. 6. These results are comparable with Fig. 2 of Ref. 4, where another method was used. The SHG curve is a result of the interplay among Fabry-Perot reflections, phase matching, and modal field profiles. As already mentioned we have immediate acces to these modal data. Moreover because there are, respectively, one and two guided modes for fundamental and second harmonic, the use of ten modes already provides accurate results. To conclude this section we note that for the sweep over the length $d$ we have to calculate the overlap integrals only once.

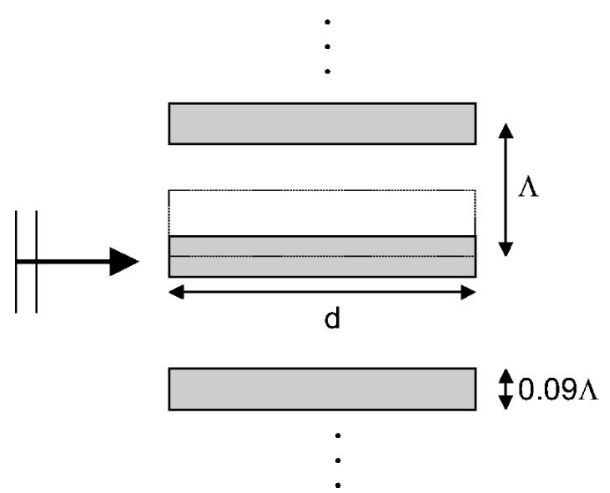

Fig. 5. Transverse grating structure. Only the section in dotted line is needed in the simulation because of symmetry.

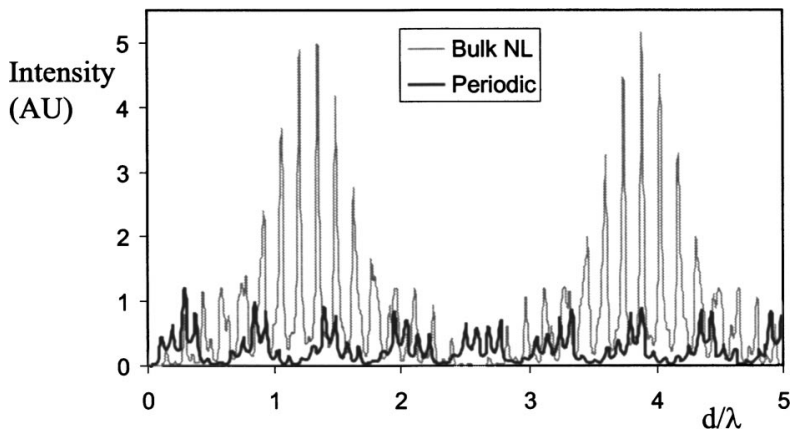

Fig. 6. Grating SHG intensity in the forward direction versus length $d$, normalized with the fundamental wavelength $\lambda$. 


\section{PHOTONIC-CRYSTAL CAVITY}

Now we study the photonic-crystal cavity device depicted in Fig. 7. In combination with the third-order Kerr nonlinearity this structure gives rise to bistable switching. ${ }^{14}$ Here we exploit the cavity effects for SHG. The device consists of a resonator mode in the center formed of a larger rod evanescently coupled to two waveguides. The left waveguide is used for input of the fundamental power. On resonance the cavity is strongly excited, and all the power is transmitted to the waveguide on the right. Off resonance the power is reflected back to the left with negligible excitation of the defect mode. In Fig. 7 the upper half shows the fundamental resonance, whereas the lower half shows the second-harmonic field on resonance.

For this device to work in a SHG setting there are conditions that have to be fulfilled for both the fundamental and second harmonic: First a bandgap and a (preferably single-mode) waveguide are necessary. Second if we demand efficient cavity SHG, there have to be resonator modes at both frequencies, preferably with good mode profile overlap. We designed such a structure, although it will prove difficult to find parameters in a real material system.

The photonic crystal used is a square lattice of square rods in air with period $a$ and diameter $d=0.5 a$. With indices $n_{\omega}=3.5$ and $n_{2 \omega}=2.95$ we can work in the first gap for the fundamental, between 0.235 and $0.314(2 \pi c / a)$, and the second gap for the second harmonic, between 0.497 and $0.569(2 \pi c / a) .{ }^{15}$ By decreasing the diameter of a line of rods to $0.3 a$ we create a single-mode waveguide for both frequencies, with normalized fundamental frequency in $[0.265,0.283]$. A second-harmonic resonance mode at $\omega_{\mathrm{sh}}^{\mathrm{res}}=0.55535(2 \pi c / a)$ is produced by a larger central rod of diameter $0.7 a$. We tune a (broader) fundamental resonance to $0.5 \omega_{\mathrm{sh}}^{\mathrm{res}}$ with a central rod index of 3.5743. Both Lorentzian transmission profiles $T_{\omega}$ and $T_{2 \omega}$ are shown in Fig. 8 with

$$
T(\omega)=\frac{\gamma^{2}}{\left(\omega-\omega^{\mathrm{res}}\right)^{2}+\gamma^{2}} .
$$

They correspond to $Q$ values of, respectively, $Q_{\omega}=301$ and $Q_{2 \omega}=3239$, with $Q=\omega^{\text {res }} / 2 \gamma$.

For the SHG calculations we assume that only the larger central rod is nonlinear, a good approximation as the field is strongest there. Because the second-harmonic defect mode is excited by the fundamental, and this mode

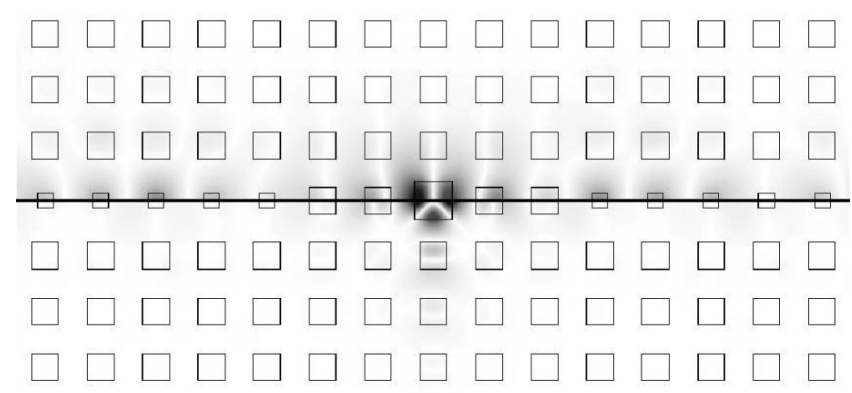

Fig. 7. Photonic crystal device geometry. The upper (lower) half shows the electric field of the fundamental (second harmonic) at resonance. Dipole and quadrupole modes are clear. Because of high $\mathrm{Q}$ the field in the lower waveguide is barely visible.

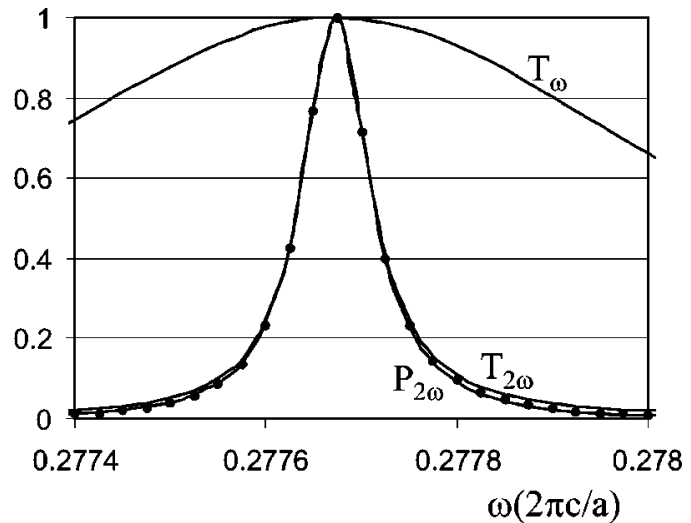

Fig. 8. Photonic crystal device results. The transmission of fundamental $T_{\omega}$ and second harmonic $T_{2 \omega}$ are shown, together with the generated second-harmonic power $P_{2 \omega}$, by curves. $P_{2 \omega}$ is normalized and all curves are plotted versus the fundamental $\omega$. Values for $T_{\omega}^{2} T_{2 \omega}$ are indicated by circles.

is equally coupled to both waveguides, the generated outgoing power to the left and the right is the same. This power is plotted in Fig. 8. Because the outgoing power is proportional to the energy of the second-harmonic mode and the second harmonic is excited by the square of the fundamental field, it results that the generation at resonance is proportional to $Q_{\omega}^{2} Q_{2 \omega}$ and the normalized SHG power profile follows $T_{\omega}{ }^{2} T_{2 \omega}$; see Fig 8 . For these calculations, only 30 modes were used for both frequencies. Moreover, if we allow the lowest ten of these modes to couple nonlinearly, we already obtain accurate results.

\section{CONCLUSIONS}

We extended the eigenmode expansion method for rigorous and efficient calculation of SHG in two-dimensional structures. By use of the undepleted-pump approximation we can describe the nonlinear sections with a linear matrix formalism. As such no grid is required in the propagation direction. A flexible coupling scheme, advanced boundary conditions, and reuse of data in periodic structures enhance the efficiency. Simulations of a photoniccrystal-cavity device demonstrate its facility for modeling complex, wavelength-scale structures. In further work we plan to examine the inclusion of pump depletion.

\section{ACKNOWLEDGMENTS}

Björn Maes and Peter Bienstman acknowledge the Flemish Fund for Scientific Research (FWO-Vlaanderen) for a doctoral and postdoctoral fellowship, respectively. Parts of this work were performed in the context of the Belgian DWTC project IAP-Photon.

Björn Maes can be reached by e-mail at bjorn.maes@intec.ugent.be.

\section{REFERENCES}

1. M. Centini, C. Sibilia, M. Scalora, G. D’Aguanno, M. Bertolotti, M. J. Bloemer, C. M. Bowden, and I. Nefedov, "Dispersive properties of finite, one-dimensional photonic 
band gap structures: applications to nonlinear quadratic interactions," Phys. Rev. E 60, 4891-4898 (1999)

2. C. De Angelis, F. Gringoli, M. Midrio, D. Modotto, J. S. Aitchison, and G. G. Nalesso, "Conversion efficiency for second-harmonic generation in photonic crystals," J. Opt. Soc. Am. B 18, 348-351 (2001).

3. Y. Dumeige, F. Raineri, A. Levenson, and X. Letartre, "Second-harmonic generation in one-dimensional photonic edge waveguides," Phys. Rev. E 68, 066617 (2003).

4. W. Nakagawa, R. C. Tyan, and Y. Fainman, "Analysis of enhanced second-harmonic generation in periodic nanostructures using modified rigorous coupled-wave analysis in the undepleted-pump approximation," J. Opt. Soc. Am. A 19, 1919-1928 (2002).

5. F. Raineri, Y. Dumeige, A. Levenson, and X. Letartre, "Nonlinear decoupled FDTD code: phase-matching in 2D defective photonic crystal," Electron. Lett. 38, 1704-1706 (2002).

6. A. Locatelli, D. Modotto, C. De Angelis, F. M. Pigozzo, and A.-D. Capobianco, "Nonlinear bidirectional beam propagation method based on scattering operators for periodic microstructured waveguides," J. Opt. Soc. Am. B 20, 1724-1731 (2001)

7. B. Shi, Z. M. Jiang, X. F. Zhou, and X. Wang, "A twodimensional nonlinear photonic crystal for strong second harmonic generation,” J. Appl. Phys. 91, 6769-6771 (2002).
8. P. Bienstman, and R. Baets, "Optical modeling of photonic crystals and VC-SELs using eigenmode expansion and perfectly matched layers," Opt. Quantum Electron. 33, 327-341 (2001).

9. B. Maes, P. Bienstman, and R. Baets, "Modeling of Kerr nonlinear photonic components with mode expansion," Opt. Quantum Electron. 36, 15-24 (2004).

10. Y. Jeong and B. Lee, "Matrix analysis for layered quasiphase-matched media considering multiple reflection and pump wave depletion," IEEE J. Quantum Electron. 35, 162-172 (1999).

11. A. Yariv, Introduction to Optical Electronics (Holt, Rinehart and Winston, 1976).

12. Camfr simulation software is freely available from http:// camfr.sourceforge.net/.

13. C. De Angelis, G. Nalesso, D. Modotto, M. Midrio, A Locatelli, and J. S. Aitchison, "Multiple-scale coupled-mode theory for second-harmonic generation in one-dimensional periodic structures," J. Opt. Soc. Am. B 20, 1853-1865 (2003).

14. M. Soljacic, M. Ibanescu, S. G. Johnson, Y. Fink, and J. D. Joannopoulos, "Optimal bistable switching in nonlinear photonic crystals,” Phys. Rev. E 66, 055601 (2002).

15. S. G. Johnson and J. D. Joannopoulos, "Block-iterative frequency-domain methods for Maxwell's equations in a planewave basis," Opt. Express 8, 173-190 (2001). 